\title{
RAMP - METERING ALGORITHMS EVALUATED WITHIN SIMPLIFIED CONDITIONS
}

\author{
Aleš JANOTA ${ }^{1,{ }^{*}}$, Peter HOLEČKO ${ }^{1}$, Michal GREGOR ${ }^{1}$, Marián HRUBOŠ ${ }^{1}$ \\ 1 Department of Control \& Information Systems, Faculty of Electrical Engineering, University of Žilina, \\ Univerzitná 8215/1, 01026 Žilina, Slovakia. \\ corresponding author: ales.janota@fel.uniza.sk.
}

\begin{abstract}
Freeway networks reach their limits, since it is usually impossible to increase traffic volumes by indefinitely extending transport infrastructure through adding new traffic lanes. One of the possible solutions is to use advanced intelligent transport systems, particularly ramp metering systems. The paper shows how two particular algorithms of local and traffic-responsive control (Zone, ALINEA) can be adapted to simplified conditions corresponding to Slovak freeways. Both control strategies are modelled and simulated using PTV Vissim software, including the module VisVAP. Presented results demonstrate the properties of both control strategies, which are compared mutually as well as with the initial situation in which no control strategy is applied.
\end{abstract}

\section{Introduction}

Majority of people live in metropolitan areas, interconnected by a network of freeways. This kind of road allows the highest speed of transport between neighbouring cities. The problem is that the freeways and their parameters are often limited by urban built-up areas, since urban planning often did not take into consideration future extension. The problem then is to increase traffic volume by construction of new traffic lanes, because there is no more space for this kind of extension. One of the possible solutions is to apply advanced intelligent transport systems and advanced technologies to make traffic flows more fluent and to increase effectiveness of freeway usage. There are various strategies ensuring balance between drivers entering the freeway and its capacity, reducing the number of traffic accidents that cause congestions and traffic delays. The best-known strategies are ramp closure (closing entries/exits), ramp metering, special use treatments, and ramp terminal treatments [1]. The authors discuss the problem of ramp metering that redistributes the freeway demand over space and time [2]. Several approaches can be used to meter ramps: with either local (isolated) or system-wide (coordinated) control; pre-timed or traffic-responsive metering [1]. Unlike the latest findings and advanced solutions that deal with various aspects of either coordinated or local control (e.g. a multi-hierarchical strategy of coordination [3], use of reinforcement learning [4], combination with route guidance [5], survey of main approaches [6], nonlinear state estimation using the revised method of numerical differentiation [7], problem of stability [8], probabilistic approach [9], etc.), this paper deals with implementation of two particular algorithms from the category of local and traffic-responsive metering only.

Most applications of ramp metering assume existence of three or more traffic lanes in one direction, when the existing infrastructure (freeway width) may not be further extended. However, the character of the freeway system in Slovakia excludes implementation of system-wide approaches. We had to choose such algorithms that do not depend so much on the number of traffic lanes and that could be used in a less developed (not so spacious) infrastructure. Therefore, we model, simulate, calibrate and evaluate two local algorithms - Zone and ALINEA algorithms that are cheap and can positively respond to the considered part of freeway system. For demonstration purposes, we have chosen the particular section of the Slovak freeway D3. Now, this section is currently under construction and contains two entries. The total length of the freeway mainline is ca. $8000 \mathrm{~m}$. The aim of the paper is to show how to adapt the two aforementioned algorithms, as considered under simplified conditions. The paper will present detailed flowcharts, and show the resulting characteristics. It will mutually compare both approaches, and also provide a comparison with the 
situation without any traffic flow control. Using data of the Slovak Road Administration (estimations from 2015), average capacity of the considered part of freeway will be somewhere between 15000 and 25000 vehicles per day. For the purpose of simulation, the following numbers of traffic volumes have been used to simulate rush hour conditions: 1050 vehicles per hour as an entry from ŽilinaStrážov to the zone which is a number close to the upper limit (i.e., 25200 vehicles per day), 100 vehicles per hour as an entry to the zone from the tunnel and 2000 vehicles per hour (exit from the zone in direction to Žilina). The numbers are somewhat higher than 2015 estimations to take permanent traffic growth into consideration.

\section{Ramp Metering}

A typical concept of the ramp metering system (one of many configurations) is shown in Fig. 1. It is necessary to remind the reader that numbers, types, and locations of detectors may vary based on different control strategies.

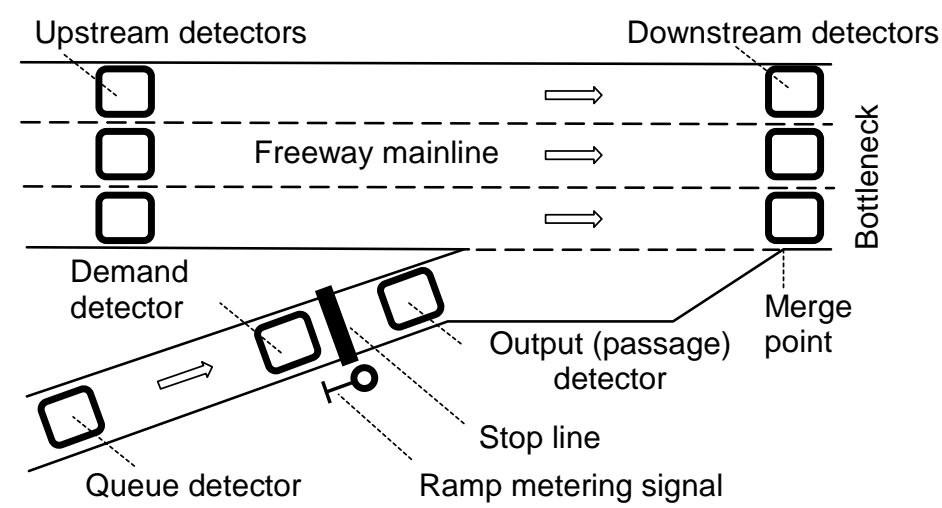

Fig. 1: Concept of ramp metering configuration.

\subsection{Zone meter algorithm}

A detailed flowchart for the implementation of the Zone algorithm is shown in Fig. 2. Due to discontinuity of the considered freeway network, the number of possible entries is low; thus, the number of sections (zones) is not suitable for the system-wide variant of the Zone algorithm. Based on the available theory, e.g., in [2], the following calculation gives a proposed rate for every meter to run in according to a percentage of $M$ :

$R_{n}=\left(M \cdot D_{n}\right) / D$,

where $R_{n}$ is the proposed rate for meter $n$ ( $n$ is a meter within the zone), $D_{n}$ is the demand for the meter $n, M$ is the metered entrance. Unlike the original version working with a group of entries, our algorithm is modified to consider only one metered ramp, i.e., we apply a different way of calculating the ramp meter rate. The aim is to ensure that the total volume exiting a zone exceeds the volume entering it. The algorithm starts with initiation, setting the signal to green and resetting the counter to 1. This first cycle will run only once at the very beginning. The next cycle counts to 60 - we read the detector values and define a new current state of the signal once per minute. The algorithm then goes on to calculate the value of the variable rmRate (ramp meter rate) based on the detector values from the entries and the exits of the zone. The variable demand is taken from the detector placed at the ramp in front of the entry to the zone. The variable queue gets the value of either 1 or 0 based on the condition of the queue detector ( 1 if a vehicle is present, 0 if no vehicle is currently detected).

After reading the needed data, internal detector memories are cleared to avoid their incremental growth. The variable capacity represents difference between optimal capacity optCap and ramp meter rate rmRate. Then the counter is cleared and set to 1 to avoid program looping. The recW (record value) commands make it possible to send actual values into Vissim programme, which is able (after setting the record table) to run simulation together with showing the current values of the variables. The remaining commands used in the algorithm set the signal. If capacity is greater or equal to demand and the current signal is not green, it will become green. If capacity is lower than demand 
when the signal is green, then it will be changed to red. If capacity is equal to demand and the signal is green, then its state will change to amber. The last condition will test for the existence of a queue. If a queue is detected and is sufficiently long, the signal immediately becomes green and the zone will empty.

The next step consists of algorithm calibration. The most critical place is the "bottleneck" placed at the first branch behind the ramp meter. Vehicles start performing various manoeuvres in order to change traffic lanes which may result in a congestion. For that reason, the optimal capacity is set to 16 , which represents 16 vehicles being allowed through the ramp meter every minute (i.e., 960 vehicles per hour). Since the algorithm is stochastic, the risk of queues appearing in this bottleneck cannot be fully eliminated.

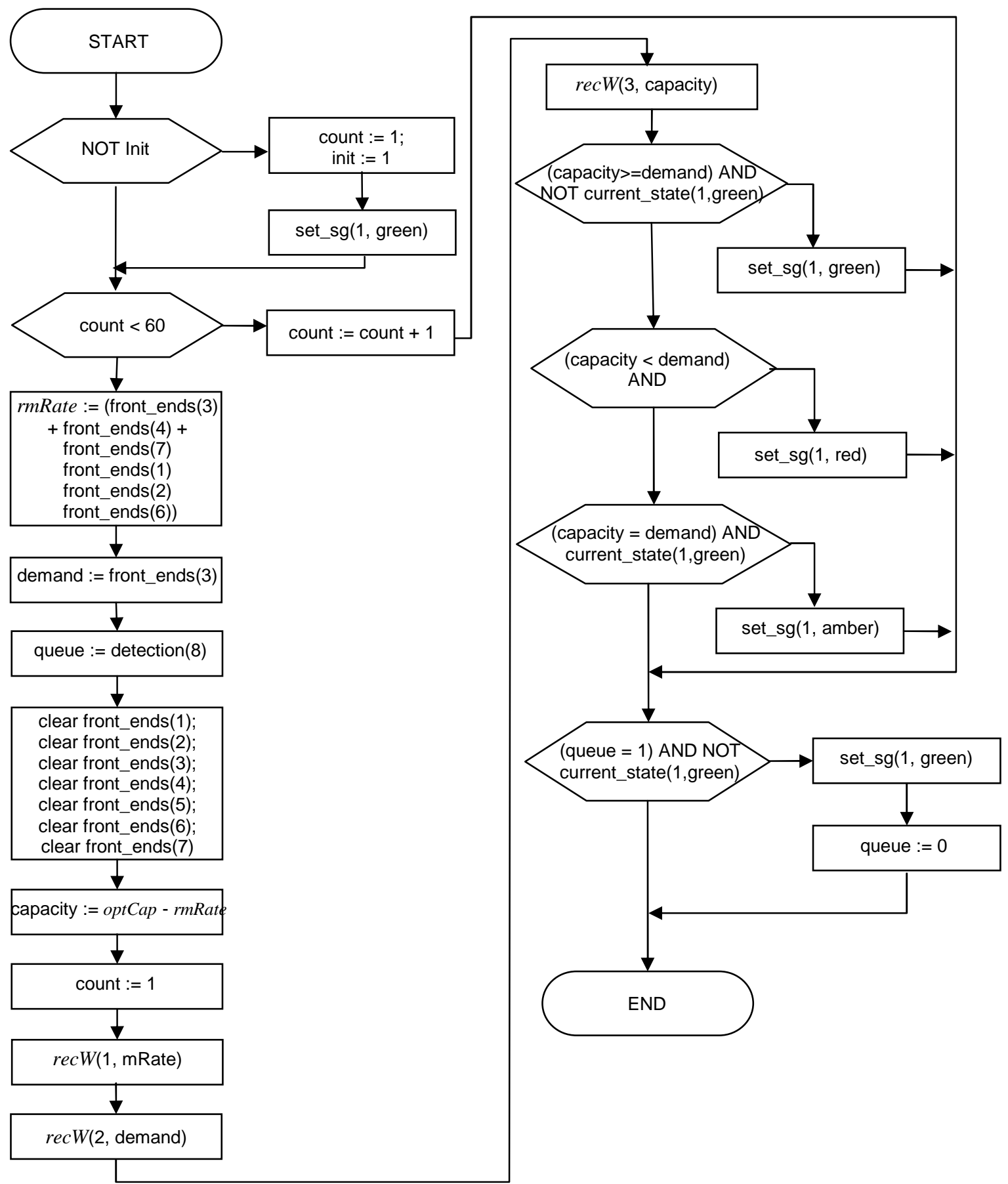

Fig. 2: Flowchart diagram of the Zone algorithm adopted for the freeway line D3, section Žilina Strážov - Żilina Brodno (Slovakia). 


\subsection{ALINEA algorithm}

The ALINEA algorithm is more complicated and its operation more thorough. The latest findings dealing with the theoretical proof of ALINEA efficiency are available either in [10] or in the case study for Istanbul freeways [11] containing simulations as well. The Zone algorithm only calculates the free capacity and compares it with the number of vehicles intending to enter the zone. The ALINEA algorithm also calculates capacity, but immediately behind the ramp metered entry, since that is the right place where traffic flow should be kept fluent. The control law of ALINEA is generally stated as:

$r(t)=r^{\prime}(t-\Delta t)+K_{R} \cdot\left(O^{*}-O(t)\right)$,

where $r(t)$ is the calculated metering rate during time interval $(t, t+\Delta t) ; \Delta t$ is the update cycle of ramp metering implementation; $r^{\prime}(t-\Delta t)$ is the measured metering rate of the time interval of $(t-\Delta t, t) ; O(t)$ is the measured occupancy of time interval $(t-\Delta t, t)$ at the downstream detector station; $k_{R}$ is a regulator parameter, used for adjusting the constant disturbances of the feedback control; $O^{*}$ is the desired occupancy at the downstream detector station.

For fluent traffic, the algorithm's interventions are minimal. To set its "aggressiveness" (increase its activity) we use the simulation constant optOccup representing the optimal occupancy. The algorithm starts with initiation, similarly as the previous one, however the signal is not set to green, but it is switched-off. The algorithm is based on the demand of vehicles intending to enter the freeway from the metered ramp. Therefore, the signal becomes active only if there is any demand and appropriate conditions behind the ramp. The variable cycle time is calculated further in the programme (in the first run, it is set to zero). It affects the duration of the red signal. If the variable time becomes equal to or greater than the cycle time, the counter is cleared. Thus, the control logic knows that the minimal duration of the signal aspect is over. The variable laneC identifying the particular traffic lane (its number) was designed to perform cyclic filling of values to the field of detectors at the freeway using the variable "number of detector" $\operatorname{det} C$. It starts with the traffic lane number 1 and goes on until the total number of lanes (laneTotal) is exceeded. In our case, the constant laneTotal is equal to 2 , since there are two lanes only, each having its own detector. The algorithm also includes a special counter to calculate the occupancy rate occRate and the total occupancy rate toccRate. The internal cycle of that counter runs once per minute, i.e., 60 cycles of the control logic. The constant $I V$ is set to the value of 1 and represents the value $(t-\Delta t)$. After reaching the value of $60 \mathrm{~s}$, the counter is cleared and the variable entry reads the value from the detector, which counts vehicles that entered through the ramp-metered entry. Then the internal memory of that detector is cleared. Within the cycle, the values of occRate and toccRate are calculated to set the minimum time of the red signal at the ramp. If the calculated value of needed cycle time is greater than or equal to $4 \mathrm{~s}$, the signal remains off. If it is lower than $4 \mathrm{~s}$ and a vehicle is detected at the entry, the condition demand is met and the cycle further verifies if time is zero or not. In case of the zero value, the signal is set to the red-amber aspect and the counter of time is cleared. In case of the value being other than zero, the algorithm finds out whether the duration of red is $3 \mathrm{~s}$ at least. If that condition is true, the signal is set to the red-amber aspect and the counter of time is cleared. Based on the previous running, the actual status of the signal is verified. If the signal was set to red-amber, the signal becomes switched-off which in fact means green (vehicles may proceed). If the signal was switched-off and the calculated time for the cycle was lower than $4 \mathrm{~s}$, the signal aspect changes to amber. Then the cycle is repeated again and the actual state of the signal changes from amber to red.

To ensure correct operation of this algorithm, its calibration is a critical issue. This kind of ramp metering is very flexible and may be focused onto various aspects such as effectiveness of capacity utilization, or dispersing platoons of vehicles so as to achieve higher gaps between vehicles. The most important aspect is the fluency of the traffic flow, here represented by the constant optOccup. If we use the ideal value recommended for three traffic lanes, in our model the effect of ramp metering will be practically eliminated, since the traffic volume will not reach values sufficient to cause a reaction of the control logic. Therefore, we had to decrease its value experimentally to 0.18 to reach the optimum.

\subsection{Implementation}

A lot of simulation tools are used to study and analyse ramp-metering problems, e.g., Quadstone Paramics, S-Paramics, AIMSUN, PTV Vissim and many others. To implement the presented algorithms, we used PTV Vissim, including the module VisVAP, which can significantly 
support design, testing, and calibration of ramp metering algorithms. Experiences with the software obtained in [12] have proved useful and valuable.

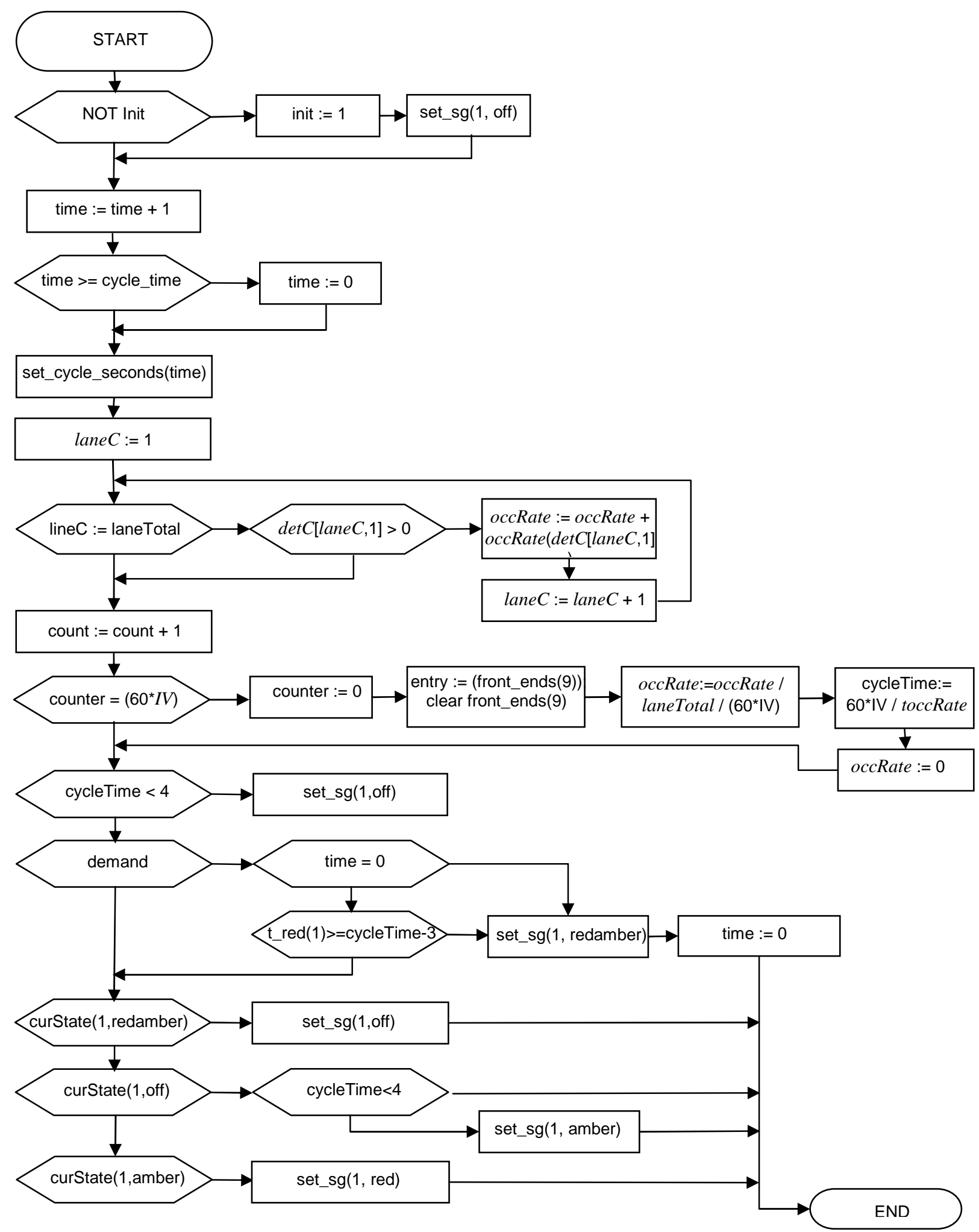

Fig. 3: Scheme diagram of the ALINEA algorithm adopted for the freeway line D3 Žilina Strážov Zilina Brodno. 
The evaluation of both algorithms was performed based on the values of average delay, average velocity and total time the vehicles spent by travelling in the model. The simulation was set to repeat 50 times with random assignment of values to random variables with the increment 1 (Fig. 4).

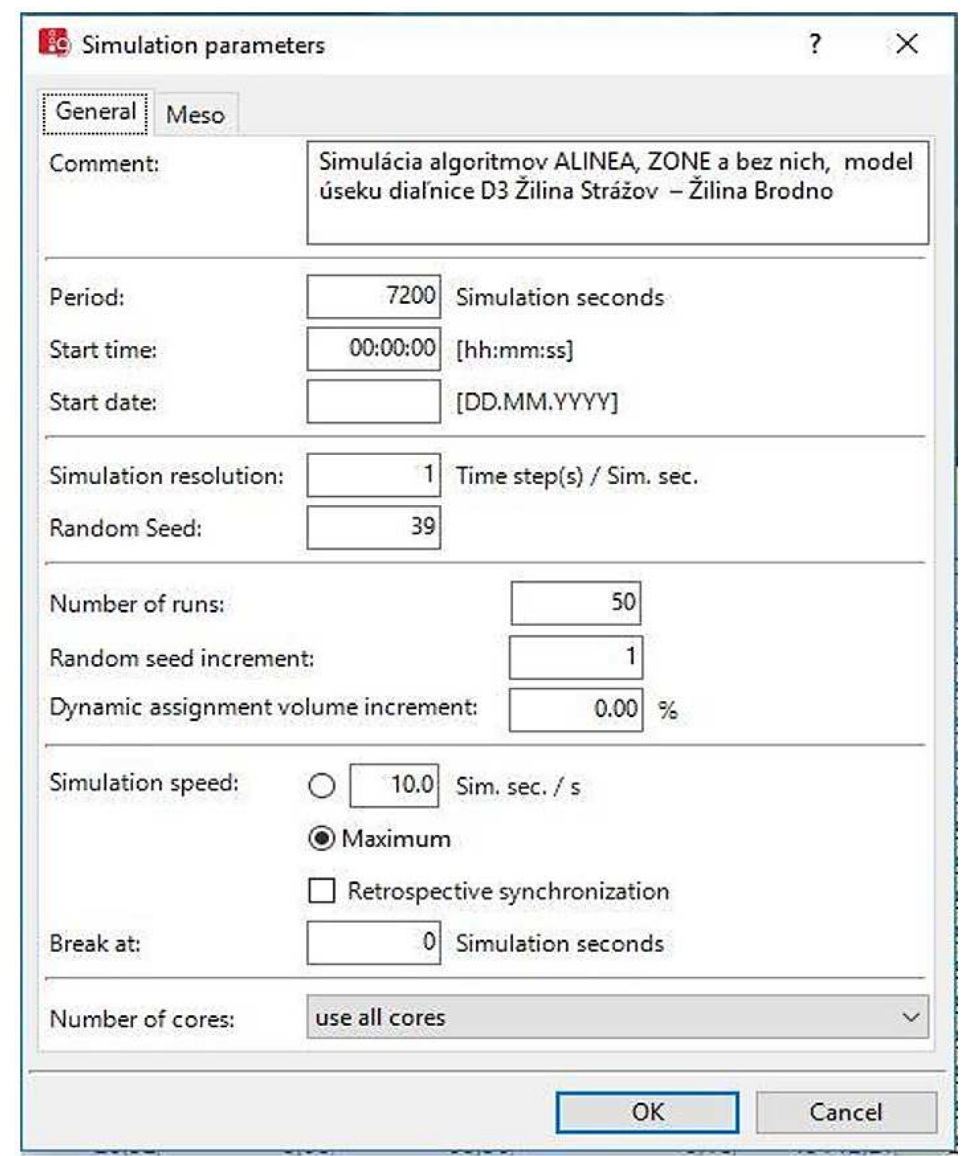

Fig. 4: Software tool PTV Vissim setting.

\section{Results}

The first criterion that was analysed was the average delay criterion, i.e., the average time by which the vehicles in the microsimulation were delayed in all simulation runs. The value was calculated as the ratio of the total delay and the number of vehicles that went through the model or found themselves in the model at the end of the simulation. This parameter seems to be the most critical one, since we require ramp-metering control to make vehicles run as fast as possible.

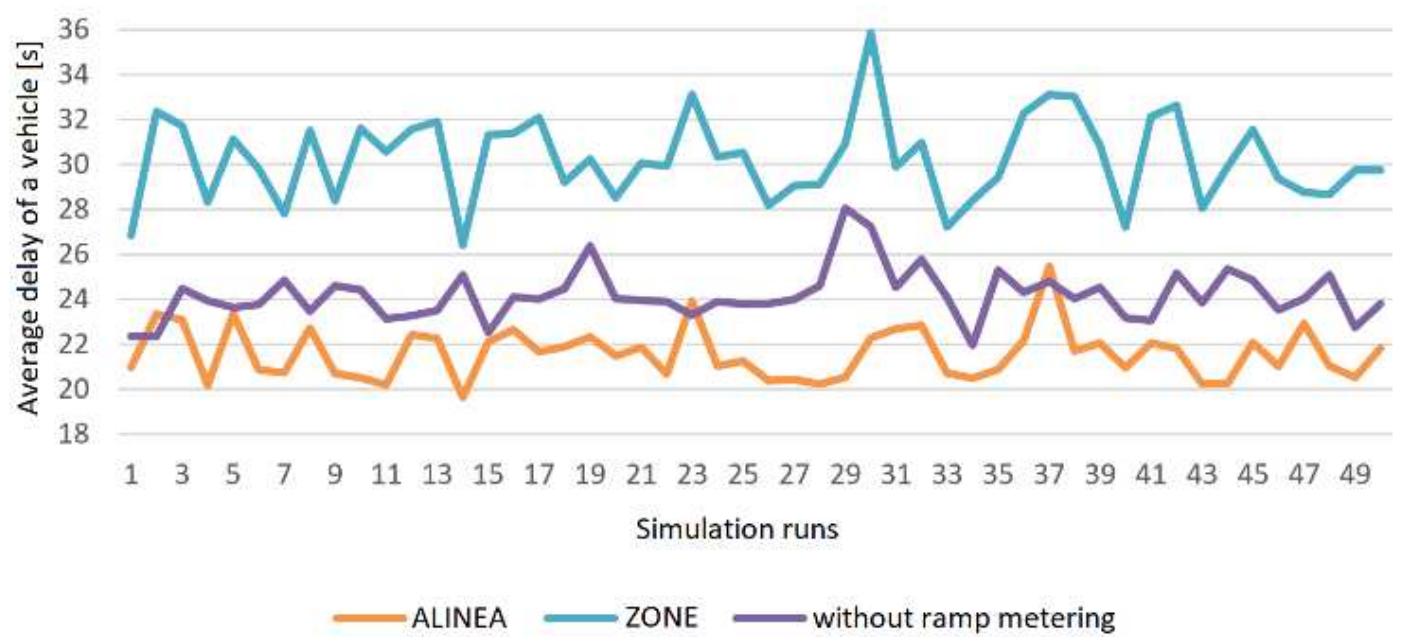

Fig. 5: Graph of average delays. 
Fig. 5 indicates that the best case occurs if the ALINEA algorithm has been implemented - the value of the delay is $21.6 \mathrm{~s}$. The worst case occurs during the use of the Zone algorithm (delay $30 \mathrm{~s}$ ), which is even worse than the no-algorithm case $(24.2 \mathrm{~s})$. That worst value is caused by relatively long delay times resulting from waiting for free capacity in the freeway mainline. On the other hand, if we evaluated utilisation of maximal capacity of the freeway and avoiding congestions, the Zone algorithm can reach better results (not covered in this paper).

Another evaluated parameter was average velocity (Fig. 6). The value was calculated as the ratio of the total distance travelled by all vehicles during simulation and the total travel time of all vehicles. With the average velocity of $59.8 \mathrm{~km} \cdot \mathrm{h}^{-1}$, the Zone algorithm brings the highest delay. The algorithm ALINEA, thanks to a well-calibrated value of optimal capacity and relatively low volume of vehicles in the simulation, causes very low delay in comparison to the no-algorithm average velocity of $60.4 \mathrm{~km} \cdot \mathrm{h}^{-1}$.

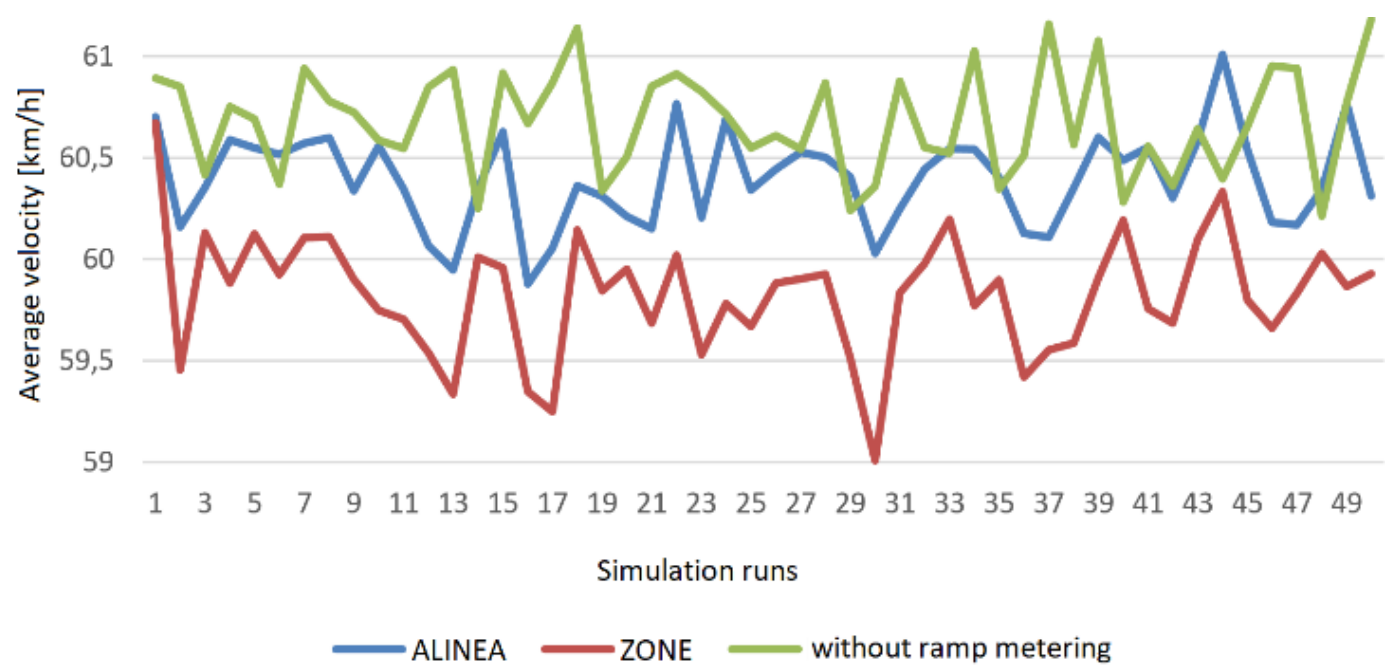

Fig. 6: Graph of average velocities.

The last presented parameter that was evaluated is total travel time (Fig. 7). The fastest drive (759 hours) can be observed for the ALINEA algorithm. The Zone algorithm and no-algorithm situations ensure similar conditions (816 hours).

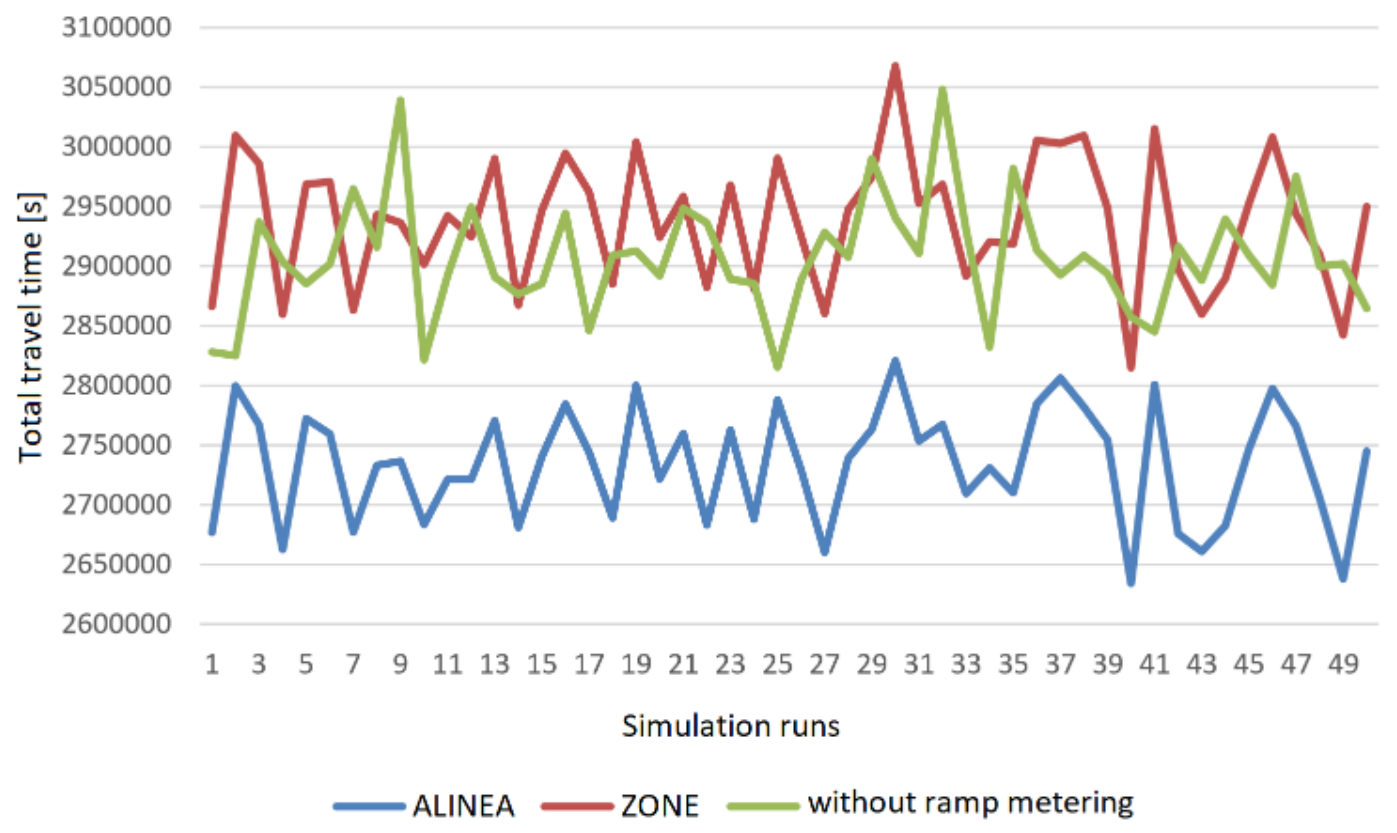

Fig. 7: Graph of a total travel time. 
To conclude, based on the findings above, the ALINEA algorithm is the most effective control strategy that makes traffic the fastest and most fluent. The bad results of the Zone algorithm very probably result from the fact that the algorithm was not originally designed to improve the evaluated parameters of freeway use.

\section{Conclusions}

Intelligent transport systems implemented into transport infrastructure can help reduce or eliminate growing congestions. Ramp metering is one of the applications that have a potential to remove ineffective utilization of freeway capacity and/or reduce unscrupulous behaviour of drivers. However, such a system must be tuned and calibrated to fulfil the given tasks and meet operators' and users' expectations. To do that, various simulation tools are available.

Two control algorithms presented in the paper showed how effective they could be if evaluated according to three different parameters. The Zone algorithm first calculates capacity and then allows vehicles to enter the zone, which results in delayed response and thus increases delay at the entry and slows the traffic. The ALINEA algorithm allows a vehicle to enter the freeway mainline and then calculates the restriction signalled at the ramp to the following vehicles. Therefore, it ensures more fluent and fast travel.

\section{Acknowledgement}

This work was supported by the project ITMS 26210120021, co-funded from EU sources and the European Regional Development Fund.

\section{References}

[1] JACOBSON, L. - STRIBIAK, J. - NELSON, L. \& SALLMAN, D.: Ramp Management and Control Handbook. FHWA-HOP-06-001, 2006.

[2] KLEIN, L.A. - MILLS, M.K. \& GIBSON, D.R.P.: Traffic Detector Handbook: Third Edition - Vol. I. FHWA-HRT-06-108, 2006.

[3] JIANG, R. - LEE, J. \& CHUNG, E.: A Multi-Hierarchical Strategy for On-Ramp Coordination. International Journal of Intelligent Transportation Systems Research, Vol. 15, Iss. 1, 2017, pp. 50-62.

[4] LU, C. - HUANG, J. - DENG. L.B. \& GONG, J.W: Coordinated Ramp Metering with Equity Consideration Using Reinforcement Learning. Journal of Transportation Engineering Part ASystems, Vol. 143, Iss. 7, 2017.

[5] PASQUALE, C. - SACONE, S. - SIRI, S. \& DE SCHUTTER, B.: A multi-class model-based control scheme for reducing congestion and emissions in freeway networks by combining ramp metering and route guidance. Transportation Research Part C-Emerging Technologies, Vol. 80, 2017, pp. 384-408.

[6] LAGEREV, R. - KAPSKI, D. - BURINSKIENE, M. \& BARAUSKAS, A.: Reducing a possibility of transport congestion on freeways using ramp control management. Transport, Vol. 32, Iss. 3, 2017, pp. 314-320.

[7] ABOUAISSA, H. - MAJID, H. \& JOLLY, D.: Nonlinear state estimation and control for freeway onramp metering. Asian Journal of Control, Vol. 19, Iss. 1, 2017, pp. 233-244.

[8] ALVAREZ-ICAZA, L. - ROSAS-JAIMES, O. \& LARRAGA, M.E.: Stability of Local On-Ramp Metering Control Laws. Asian Journal of Control, Vol. 19, Iss. 2, 2017, pp. 494-509.

[9] MEHR, N. \& HOROWITZ, R.: Probabilistic Freeway Ramp Metering. Proc. of the ASME $9^{\text {th }}$ annual dynamic systems and control conference, Vol. 2, 2017.

[10] ABOUAISSA, H. - FLIESS, M. \& JOIN, C.: On ramp metering: towards a better understanding of ALINEA via model-free control. International Journal of Control, Vol. 90, Iss. 5, 2017, pp. 10181026.

[11] ABUAMER, I.M. \& CELIKOGLU, H.B.: Local Ramp Metering Strategy ALINEA: Microscopic Simulation Based Evaluation Study on Istanbul Freeways. $19^{\text {th }}$ Euro Working Group on Transportation Meeting (EWGT2016), Vol. 22, 2017, pp. 598-606.

[12] SRNKA, T.: Control of the Vehicles Flow in the "Ramp Metering" Application (in Slovak). MSc. thesis, No. 28260220172022 , KRIS EF UNIZA Žilina, 2017. 